\title{
Age-related neural differences in affiliation and isolation
}

\author{
Janelle N. Beadle • Carolyn Yoon • Angela H. Gutchess
}

Published online: 28 February 2012

(C) Psychonomic Society, Inc. 2012

\begin{abstract}
While previous aging studies have focused on particular components of social perception (e.g., theory of mind, self-referencing), little is known about age-related differences specifically for the neural basis of perception of affiliation and isolation. This study investigates age-related similarities and differences in the neural basis of affiliation and isolation. Participants viewed images of affiliation (groups engaged in social interaction) and isolation (lone individuals), as well as nonsocial stimuli (e.g., landscapes), while making pleasantness judgments and undergoing functional neuroimaging (BOLD fMRI). Results indicated age-related similarities in response to affiliation and isolation in recruitment of regions involved in theory of mind and self-referencing (e.g., temporal pole, medial prefrontal cortex). Yet age-related differences also emerged in response to affiliation and isolation in regions implicated in the theory of mind, as well as self-referencing. Specifically, in response to isolation versus affiliation images, older adults showed greater recruitment than did younger adults of the temporal pole, a region that is important for retrieval of personally relevant memories utilized to understand others' mental states. Furthermore, in response to images of affiliation versus isolation, older adults showed greater
\end{abstract}

J. N. Beadle • A. H. Gutchess

Department of Psychology, Neuroscience Program,

Brandeis University,

Waltham, MA, USA

C. Yoon

Marketing Department, Stephen M. Ross School of Business, University of Michigan,

Ann Arbor, MI, USA

J. N. Beadle $(\square)$

Department of Psychiatry, University of Iowa

Roy J. \& Lucille A. Carver College of Medicine,

200 Hawkins Dr., W-278 GH,

Iowa City, IA 52242, USA

e-mail: janelle-beadle@uiowa.edu recruitment than did younger adults of the precuneus, a region implicated in self-referencing. We suggest that age-related divergence in neural activation patterns underlying judgments of scenes depicting isolation versus affiliation may indicate that older adults' theory of mind processes are driven by retrieval of isolation-relevant information. Moreover, older adults' greater recruitment of the precuneus for affiliation versus isolation suggests that the positivity bias for emotional information may extend to social information involving affiliation.

Keywords Aging · Social $\cdot$ Neuroimaging ·

Self-referencing $\cdot$ Theory of mind

Aging produces many cognitive, emotional, and neural changes that impact social perception. Older adults prioritize affiliating with close others and are motivated to maintain high-quality and rewarding social relationships, as purported by socio-emotional selectivity theory (Carstensen, Isaacowitz, \& Charles, 1999). Yet social isolation may also be an important factor in aging, because older adults are more likely than younger adults to experience time alone, due to the loss of spouses or friends as they age (Kaplan \& Kronick, 2006). Despite the central importance of social perception in aging, most studies of the neural basis of socio-emotional perception have focused on basic emotions (Gunning-Dixon et al., 2003; Mather et al., 2004; Tessitore et al., 2005), while social perception has been largely neglected. Some aging studies nonetheless are informative about components that are critical for social perception, such as theory of mind (Castelli et al., 2010) and self-referencing (Gutchess, Kensinger, \& Schacter, 2007, 2010). The present study extends this prior work to investigate the neural basis of age-related differences in the perception of affiliation and isolation.

First, we operationally define affiliation and isolation. Specifically, affiliation entails interacting with others, while 
isolation involves being alone. A need to affiliate has been defined as a desire to nurture positive relationships with others (Koestner \& McClelland, 1992). Isolation, on the other hand, may be an involuntary state whereby an individual is excluded from a social group. This study focuses on understanding age-related differences in perceiving affiliation and isolation by investigating specific components of social perception in younger and older adults that have been characterized in prior studies - theory of mind and self-referencing.

Perception of affiliation or isolation is expected to recruit neural regions involved in theory of mind in younger and older adults. Key regions involved in theory of mind include the temporal pole and the medial prefrontal cortex (among others), which are engaged through structured theory of mind tasks where the goal is to assess others' mental states (Frith \& Frith, 2003). Viewing scenes or videos of others socially interacting, which can be characterized as affiliation, also engages theory of mind regions in younger adults (Iacoboni et al., 2004; Wagner, Kelley, \& Heatherton, 2011). Furthermore, greater activity in theory of mind regions when viewing pictures of others involved in social interaction is associated with greater self-reported dispositional empathy (Wagner et al., 2011). Perceiving isolation also recruits theory of mind regions in younger adults, as can be seen when individuals observe others being socially excluded or isolated (Masten, Morelli, \& Eisenberger, 2011). Therefore, perceiving scenes of both affiliation and isolation recruits brain regions involved in understanding others' mental states in younger adults.

In aging, theory of mind ability is reduced (Maylor, Moulson, Muncer, \& Taylor, 2002; Slessor, Phillips, \& Bull, 2008; Sullivan \& Ruffman, 2004). However, few studies have examined the neural underpinnings of this change in theory of mind ability with aging. Of these studies, agerelated differences have emerged in brain regions involved in understanding others' mental states, where older adults have shown greater activity in the supplementary motor region (BA 6) and the inferior frontal gyrus (BA 44; Castelli et al., 2010) than have younger adults. These specific regions have been found to play a role in experiencing others' thoughts and emotions as a means by which to understand them, which is thought to occur through neural systems that respond to one's own and others' actions (Rizzolatti \& Craighero, 2004). Yet age-related preservation has also been found in the recruitment of the temporal pole (Castelli et al., 2010), a region thought to be involved in retrieving relevant personal memories and social scripts used to understand the thoughts and feelings of others (Frith \& Frith, 2003; Moriguchi et al., 2006). Nevertheless, behaviorally, it has been shown that there are some age-related differences in terms of memory retrieval for different types of social perception (Gutchess, Yoon, \& Schultheiss, 2012).
Specifically, older adults have shown better memory for visual scenes depicting isolation than for those depicting affiliation, whereas younger adults remember both types to a similar extent (Gutchess et al., 2012). We reason that this behavioral effect is likely to be manifested in terms of the neural recruitment of the temporal pole, because of the involvement of this region in memory retrieval relevant to social situations. Thus, we predict that older adults will show greater recruitment of the temporal pole region in response to isolation scenes than in response to affiliation scenes.

Self-relevant information is preferentially encoded relative to information that does not involve the self. Selfreferencing is defined as detecting whether environmental information is self-relevant, such as judging how well a personality trait (e.g., hard-working) describes oneself or others (Gutchess et al., 2007; Kelley et al., 2002; Macrae, Moran, Heatherton, Banfield, \& Kelley, 2004). Several studies have shown that self-relevant personality trait information is better remembered than that same information about another person (Gutchess et al., 2007; Kelley et al., 2002). Furthermore, a recent study showed that adopting a self-relevant viewpoint increased accuracy and decreased reaction time, relative to a third-person viewpoint, in a simple counting task (Vogeley et al., 2004). In terms of the neural mechanisms of this process, a meta-analysis of tasks assessing self-referencing revealed the recruitment of three key neural regions: the precuneus/posterior cingulate cortex, the ventromedial prefrontal cortex/pre- and subgenual anterior cingulate cortex, and the dorsomedial prefrontal cortex/ supragenual anterior cingulate cortex (Northoff et al., 2006). As a whole, these studies suggest preferential processing of self-relevant information in social contexts that recruit a circuit of cortical midline regions.

With age, self-referencing shows relative preservation, both behaviorally and neurally, of key regions involved in this process (Gutchess et al., 2007). Older adults also show a preference toward memory for positive self-relevant information over negative (Gutchess et al., 2007; Kensinger \& Schacter, 2008; Leclerc \& Kensinger, 2008; reviewed in Kensinger \& Leclerc, 2009). Specifically, older adults show greater activity in regions involved in self-referencing, such as the ventromedial prefrontal cortex, toward positive than toward negative images, with the reverse pattern being seen in younger adults (Leclerc \& Kensinger, 2008). Furthermore, in response to accurate memory for positive objects, older adults showed greater recruitment of the medial prefrontal cortex and cingulate gyrus than did younger adults (Kensinger \& Schacter, 2008), two regions implicated in self-referencing. These results are in line with research on the positivity effect in aging, whereby older adults show greater responsiveness to positive than to negative material, relative to younger adults (Mather \& Carstensen, 2005). Memory for positive self- 
relevant information is most similar to the affiliation condition investigated in the present study, because affiliation is typically thought to involve positive interactions with others, such as cooperation (Bora, Yucel, \& Allen, 2009). Older adults are motivated to affiliate with close others in order to maintain quality and rewarding relationships (Carstensen et al., 1999). On the other hand, isolation is more often associated with negative social experiences, such as loneliness or social rejection. Thus, we predict that older adults will show greater recruitment of neural regions involved in self-referencing (e.g., the precuneus and medial prefrontal cortex) in response to affiliation scenes than in response to isolation scenes.

The present study extends the literature by specifically investigating the neural basis of age-related differences in the perception of affiliation and isolation. On the basis of the aforementioned findings, we hypothesize that when perceiving social interactions depicting affiliation or isolation, older and younger adults will recruit regions involved in theory of mind (e.g., the temporal pole) and self-referencing (e.g., the precuneus and medial prefrontal cortex). We predict that older adults will show greater recruitment of self-referential regions (e.g., the precuneus, medial prefrontal cortex) than will younger adults in response to perceiving affiliation than in response to isolation, on the basis of prior results suggesting that older adults exhibit a preference for positive selfreferential information (Gutchess et al., 2007; Kensinger \& Schacter, 2008; Leclerc \& Kensinger, 2008). To corroborate previous studies of theory of mind in aging (Castelli et al., 2010), we further predict that older adults will show greater recruitment of regions in the theory of mind network involved in experiencing others' mental states as if they were one's own (e.g., the supplementary motor region, inferior frontal cortex). Finally, we hypothesize that older adults will show greater recruitment of a specific theory of mind region - the temporal pole, involved in utilizing personal memories to understand others' minds - in response to isolation than in response to affiliation. This prediction is based on prior behavioral findings that older adults show enhanced memory for isolation scenes, as compared with affiliation scenes (Gutchess et al., 2012).

\section{Method}

\section{Participants}

Sixteen younger adults ( $M_{\text {age }}=20.75$ years, $S D=1.44$ ) and 16 older adults $\left(M_{\text {age }}=70.31\right.$ years, $\left.S D=5.04\right)$ participated in the study in exchange for payment. Participants had to be righthanded, speak English as a native language, and be in good neurological, psychological, and physical health, as well as be free of medications that affect the central nervous system and other contraindications for MRI scanning. Each age group included 9 females and 7 males. Older adults reported higher education levels than did younger adults (see Table 1), which is consistent with the fact that the latter group comprised primarily undergraduate students. Written informed consent, approved by the University of Michigan Institutional Review Board, was obtained from all study participants.

Neuropsychological testing revealed age-related similarities and differences that are consistent with previous aging research (see Table 1). Assessment of cognitive functions included working memory (WAIS-III digit span forward and backward, letter-number sequencing; Wechsler, 1997), processing speed (digit comparison task; Hedden et al., 2002; for reviews, see Salthouse, 1996, 2000), vocabulary (Shipley, 1986), and a screening tool for cognitive orientation (Mini-Mental State Exam [MMSE]; Folstein, Folstein, \& McHugh, 1975). Despite the small but significant age difference on the MMSE, both age groups were above the orientation cutoff score of 26, with mean scores around 29 (out of 30). Self-rated health was measured in comparison with peers with the item, "Compared to other people your own age, how would you rate your physical health?" Participants rated this item on a 5-point scale, with a rating of 3 indicating average and 4 denoting better than average health. Older and younger adults did not differ in their self-reports of physical health (see Table 1).

\section{Materials}

The social perception task entailed rating the pleasantness of color images that depicted either social information (affiliation or isolation) or nonsocial information (serving as the control condition; see Fig. 1 for examples). The social affiliation condition included images depicting more than one individual, typically interacting in a group setting. In contrast, the social isolation condition included images of lone individuals in the context of a scene. These social scenes were selected to depict a variety of types of interactions. For example, affiliation pictures depicted participants in a business or social setting or walking side by side down the street but did not uniformly depict individuals as happy to be in the presence of others. For the isolation pictures, although individuals were depicted alone within scenes, the context did not suggest that they were being excluded by others. The nonsocial control condition included images of objects and naturalistic scenes that were selected to correspond to the configuration of social scenes (e.g., a cluster of three chairs in a group or a parking lot filled with cars vs. a lone tree presented against a background). The pictures were chosen on the basis of the fact that they had similar properties, such as the number of objects represented in each image (e.g., nonsocial scenes contained some collections of objects, like the affiliation pictures that had multiple individuals, and single objects, like the isolation 
Table 1 Neuropsychological characteristics across age groups

\begin{tabular}{llll}
\hline & Younger & Older & $p$-value \\
\hline Age & $20.75(1.44)$ & $70.31(5.04)$ & 9 females \\
Gender & 9 females & $16.83(2.01)$ & $<.001$ \\
Years of education & $14.38(1.18)$ & $4.20(.56)$ & .001 \\
Self-rating of health & $4.00(.63)$ & $59.88(10.07)$ & .36 \\
Digit comparison & $78.75(11.59)$ & $11.31(1.96)$ & .57 \\
Digit span (forward) & $10.94(1.77)$ & $7.81(2.20)$ & .93 \\
Digit span (backward) & $7.75(1.91)$ & $9.69(1.99)$ & .02 \\
Letter-number sequencing & $12.06(3.26)$ & $36.69(2.91)$ & .001 \\
Shipley Vocabulary & $32.00(2.50)$ & $29.13(.72)$ & \\
MMSE & $29.75(.45)$ & & .006 \\
\hline
\end{tabular}

pictures). In terms of the ages of the individuals in the stimuli, the pictures were chosen to represent a range of ages that were appropriate for both younger and older adults.

\section{Procedure}

The social perception task was presented using E-Prime software (Psychology Software Tools, Inc.), which interfaced with the scanner using the IFIS 9.0 system (MRI Devices Corp.) The display was presented via goggles (Resonance Technology VisuaStim XGA). Participants read instructions on the screen and were asked to respond as quickly but as accurately as possible. The social perception task consisted of two functional neuroimaging runs, with each run lasting $6 \mathrm{~min}$. Across the two runs, participants viewed a total of 90 pictures, with equal numbers of affiliation, isolation, and nonsocial pictures. There were two counterbalanced versions of the stimuli across participants, and the trials were randomly ordered and interspersed with fixation crosses in a jittered design (Dale, 1999). Pictures were presented for $4 \mathrm{~s}$, during which the participant rated the pleasantness of the image on a 5-point scale by pressing the button corresponding to their rating: very pleasant (thumb), pleasant (index finger), neutral

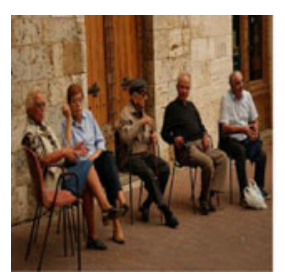

Affiliation

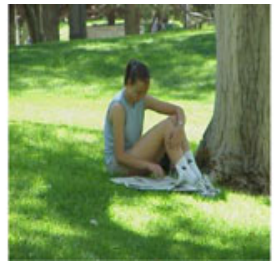

Isolation

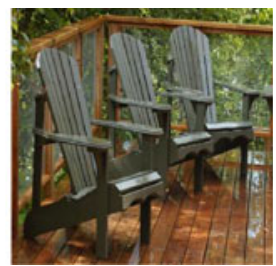

Nonsocial
Fig. 1 Participants viewed and made ratings of pleasantness for three different stimuli conditions: affiliation (images of groups of individuals interacting; left), isolation (images of a lone individual; center), or nonsocial (images of objects and landscapes; right) (middle finger), unpleasant (ring finger), and very unpleasant (pinky finger). ${ }^{1}$

Image acquisition and analysis

Image acquisition A 3T GE LX scanner (General Electric, Milwaukee, WI) was used to acquire 180 TRs (TR = $2,000 \mathrm{~ms}, \mathrm{TE}=30 \mathrm{~ms}, \mathrm{FA}=90^{\circ}$, a FOV of $22 \mathrm{~cm}$, and a $64 \times 64$ matrix) in each of two runs. Twenty-nine contiguous slices, $4 \mathrm{~mm}$ thick, were collected with a forward and reverse spiral sequence. A high-resolution SPGR structural image also was acquired. Image preprocessing included slice-time correction with an 8-point Hanning windowed sinc interpolation implemented in $\mathrm{C}++$, motion correction using AIR 3.08 (Woods, Cherry, \& Mazziotta, 1992), normalization to a Montreal Neurological Institute Template (MNI), resampling to $2-\mathrm{mm}$ cubic voxels, and spatial smoothing to a 6-mm full-width half-maximum isotropic Gaussian kernel. The SPM2 software program (Wellcome Department of Cognitive Neurology, London, U.K.) was used for the latter preprocessing stages and data analysis.

Image analysis The data analysis included an event-related design comparing older and younger adults' responses to social versus nonsocial information, as well as the direct comparison of the two types of social information (affiliation vs. isolation). There were three regressors of interest in the general linear model, which included social affiliation,

\footnotetext{
${ }^{1}$ Participants rated the pleasantness of these images on a 5-point scale, from $1=$ very pleasant to $5=$ very unpleasant. Although the average pleasantness ratings for all conditions fell between the pleasant and neutral portions of the scale, ratings differed across conditions, $F(2,58)=32.62$, $p<.001, \eta_{\mathrm{p}}{ }^{2}=.53$, with pictures of social affiliation rated as significantly more pleasant $(M=2.14)$ than control pictures $(M=2.46), t(30)=3.72, p<$ .002 . Control pictures were also rated as more pleasant than pictures of social isolation $(M=2.73), t(30)=4.23, p<.001$. No main effects or interactions with age approached significance $\left(F_{\mathrm{S}}<1\right)$. Data from 1 older adult were lost due to a program error.
} 
social isolation, and nonsocial (control) regressors for each of the two runs. Session regressors were also included for each of the two runs. Each event was convolved with a canonical hemodynamic response function. For betweengroup comparisons, contrast images were smoothed with an $8-\mathrm{mm}$ full-width half-maximum isotropic Gaussian kernel for a total of $10 \mathrm{~mm}$ of smoothing across the two stages. A random effects group analysis was used to compare age differences in brain activity in response to the conditions of interest (social affiliation, social isolation, and nonsocial), using two-sample $t$-tests with a threshold of $p<.001$ (uncorrected) and an extent threshold of 5 voxels. To assess regions of activity in common for younger and older adults, first a mask of voxels significant at $p<.01$ with no voxel extent threshold was created for one group or condition and was used to test for overlapping regions of activation in the second group/condition, for a conjoint probability of $p<$ .0001, using Fisher's method (Fisher, 1950; Lazar, Luna, Sweeney, \& Eddy, 2002). This method was also used to determine age similarities in response to affiliation in comparison with isolation.

We first compared regions responding to social (affiliation and isolation) pictures, as compared with nonsocial pictures, testing the following contrast: (Affiliation + Isolation) -
Nonsocial. Commonalities and differences across the age groups were then tested in these regions, as well as those age differences that extended across affiliation and isolation pictures, using the conjunction approach described above. Age groups were then compared on the activity selectively associated with affiliation versus isolation, using masks to assess the conjunction of the two groups for the commonality analyses. In order to elucidate age differences, we conducted contrasts of [Older (Affiliation - Isolation) - Younger (Affiliation - Isolation)] and [Younger (Affiliation - Isolation) Older (Affiliation - Isolation)]. Results of these contrasts were further clarified by region of interest (ROI) analyses that indicated the response of older, as compared with younger, adults in a particular region in response to affiliation versus isolation pictures. ROIs are presented only to characterize the patterns of results as revealed by the contrasts; because they are not independent analyses, they are not used to identify additional regions in which the age groups differ (e.g., Vul, Harris, Winkielman, \& Pashler, 2009). We extracted the percentage of signal change from the ROI using MarsBaR software (Brett, Anton, Valabregue, \& Poline, 2002). These estimates were extracted from spheres with a 6-mm radius, centered around the peak coordinates, and averaged across the 3 rd-7th time points.

Table 2 Age similarities in social perception

\begin{tabular}{|c|c|c|c|c|c|c|}
\hline Region & $\mathrm{BA}$ & \multicolumn{3}{|c|}{ Activation Peak $(\mathrm{x}, \mathrm{y}, \mathrm{z})$} & \# voxels & $t$ value \\
\hline \multicolumn{7}{|c|}{ Social - Control (Conjunction Older and Younger Adults) } \\
\hline $\mathrm{R}$ middle temporal & 37 & 52 & -62 & 6 & 6,852 & 10.90 \\
\hline $\mathrm{R}$ middle temporal & 21 & 54 & -44 & -4 & & 9.85 \\
\hline $\mathrm{R}$ inferior occipital & 19 & 48 & -82 & -8 & & 9.13 \\
\hline L middle temporal & 37 & -50 & -68 & 6 & 6,155 & 8.45 \\
\hline L occipito-temporal & $37 / 19$ & -50 & -86 & -6 & & 5.85 \\
\hline $\mathrm{L}$ middle temporal & 21 & -52 & -52 & 6 & & 5.10 \\
\hline $\mathrm{R}$ precuneus & 7 & 4 & -62 & 34 & 1,396 & 7.47 \\
\hline L precuneus & 7 & -6 & -56 & 42 & & 6.06 \\
\hline $\mathrm{R}$ inferior temporal & 37 & 46 & -50 & -24 & 535 & 6.08 \\
\hline $\mathrm{R}$ medial prefrontal & 10 & 4 & 56 & 26 & 2,301 & 5.91 \\
\hline L medial prefrontal & 9 & -12 & 60 & 30 & & 4.39 \\
\hline L medial prefrontal & 9 & -4 & 50 & 44 & & 4.36 \\
\hline $\mathrm{R}$ amygdala & & 16 & -2 & -16 & 247 & 5.42 \\
\hline $\mathrm{L}$ inferior frontal & 45 & -52 & 22 & 10 & 58 & 4.84 \\
\hline L amygdala & & -26 & -4 & -16 & 182 & 4.62 \\
\hline $\mathrm{R}$ middle temporal & 20 & 58 & -10 & -20 & 251 & 4.21 \\
\hline $\mathrm{R}$ temporal pole & 38 & 56 & 8 & -32 & & 3.12 \\
\hline L temporal pole & 38 & -50 & 10 & -38 & 617 & 4.17 \\
\hline L temporal pole & 38 & -42 & 24 & -34 & & 4.01 \\
\hline L temporal pole & 38 & -30 & 20 & -30 & & 3.44 \\
\hline
\end{tabular}

Note. The data show those regions that emerge in the contrast for both younger and older adults, with an overall threshold of $p<.0001$ with an extent threshold of 5 voxels. Up to three local maxima, separated by at least $8 \mathrm{~mm}$, are displayed 


\section{a}
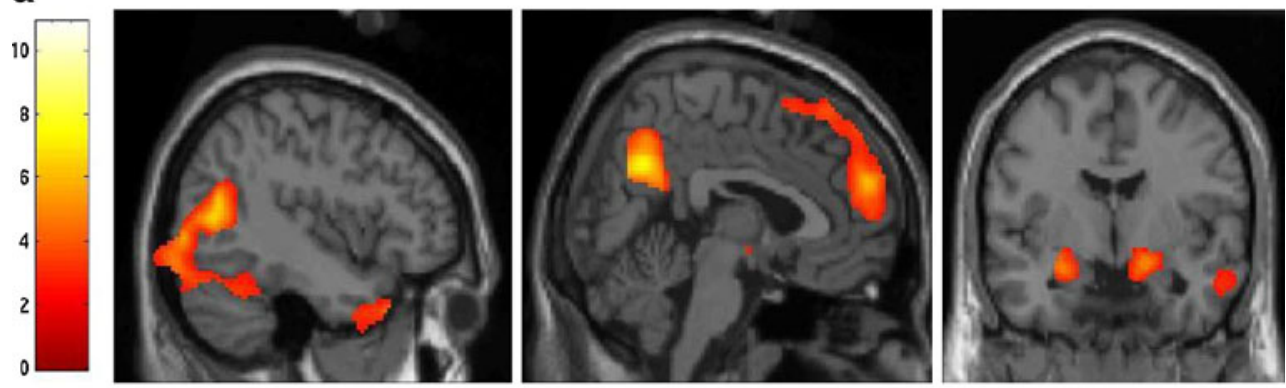

b
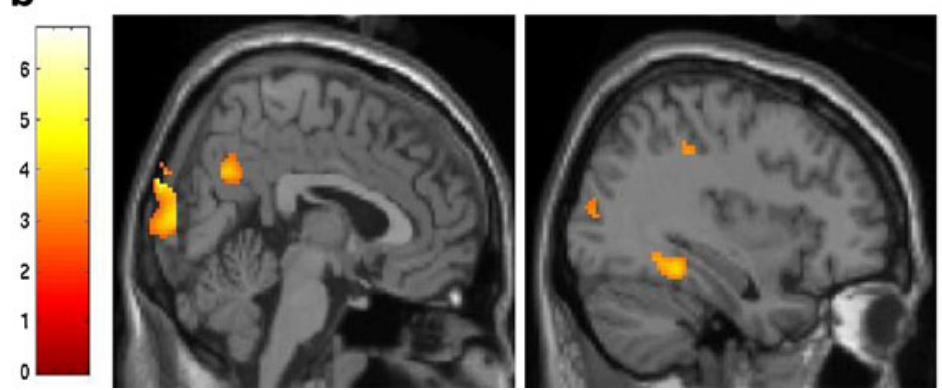

Fig. 2 a A conjunction analysis determined age similarities, or regions of shared activation, between younger and older adults when perceiving social as compared with nonsocial scenes. Social perception produced greater activity in the temporal pole (left), the medial prefrontal cortex (center), and the amygdala (right). b A conjunction analysis determined neural regions of shared activation between younger and older adults in response to affiliation, in comparison with isolation. Perception of affiliation produced greater activity in the precuneus (left), while perception of isolation produced greater activity in superior/inferior parietal regions (right)

\section{Results}

Functional magnetic resonance imaging data

Similarities with age in the perception of social versus nonsocial scenes In the conjunction analysis of younger and older adults, perception of social scenes, relative to nonsocial scenes, produced greater brain activity in the temporal pole and medial prefrontal cortex (see Table 2, Fig. 2a), regions implicated in theory of mind and selfreferencing, as well as the amygdala (see Table 2, Fig. 2a). A conjunction analysis determined shared brain regions across younger and older adults that responded more to affiliation than to isolation or vice versa (see Table 3, Fig. 2b). Affiliation produced a greater response than did isolation in the precuneus (see Table 3, Fig. 2b), a region implicated in self-referencing and theory of mind. Isolation, relative to affiliation, elicited a greater response in the superior/inferior parietal regions (see Table 3, Fig. 2b), which are involved in comprehending others' intentions. In addition, isolation elicited greater activity in a distinct peak in the precuneus region (see Table 3). The recruitment of the precuneus for both affiliation and isolation is further clarified by age group differences found in this region that differ as a function of the type of social interaction perceived (described in the next section).
Age-related differences in the perception of social versus nonsocial scenes While the conjunction analyses assessed age-related similarities in response to social versus nonsocial perception, the next series of analyses investigated agerelated differences by contrasting older and younger adults' neural responses to social versus nonsocial information. We used a conjunction analysis to investigate brain activity that was shared in response to both affiliation and isolation, in comparison with nonsocial stimuli, and then contrasted the age groups (Table 4, Fig. 3a). Although not specifically hypothesized a priori, younger adults showed greater activity than did older adults in response to both affiliation and isolation scenes (relative to nonsocial scenes) in the hippocampus (see Table 4, Fig. 3a), which is known to play a critical role in encoding information into memory. No brain regions showed significantly greater brain activation for older adults, as compared with younger adults, in response to both types of social scenes versus nonsocial scenes.

Next, age differences in the neural response to affiliation and isolation were directly compared (see Table 4, Fig. 3b, c). A random effects analysis compared the neural response to affiliation and isolation between older and younger adults via the following contrast: [Older (Affiliation - Isolation) - Younger (Affiliation - Isolation)]. Those regions that showed statistically significant age differences were then further examined through an ROI analysis using MarsBaR to assess the percent 
Table 3 Age similarities in social perception in response to affiliation versus isolation

\begin{tabular}{|c|c|c|c|c|c|c|}
\hline \multirow{2}{*}{$\frac{\text { Region }}{\text { A. Affiliation - Isolation }}$} & \multirow[t]{2}{*}{$\mathrm{BA}$} & \multicolumn{3}{|c|}{ Activation Peak $(\mathrm{x}, \mathrm{y}, \mathrm{z})$} & \multirow[t]{2}{*}{ \# voxels } & \multirow[t]{2}{*}{$t$ value } \\
\hline & & & & & & \\
\hline $\mathrm{R}$ cuneus & $17-19$ & 10 & -94 & 24 & 1,553 & 6.8 \\
\hline L superior occipital & 17 & -8 & -100 & 8 & & 6.18 \\
\hline L lingual gyrus & 17 & -2 & -104 & -6 & & 4.14 \\
\hline $\mathrm{n} / \mathrm{a}$ & & -44 & -88 & -8 & 363 & 4.89 \\
\hline $\mathrm{n} / \mathrm{a}$ & & -50 & -82 & 0 & & 3.42 \\
\hline L lingual gyrus & 19 & -34 & -84 & -14 & & 3.42 \\
\hline $\mathrm{R}$ precuneus & 7 & 4 & -64 & 32 & 168 & 3.98 \\
\hline $\mathrm{R}$ middle temporal & 37 & 60 & -62 & 12 & 593 & 3.97 \\
\hline $\mathrm{R}$ lateral occipital gyrus & $37 / 19$ & 56 & -74 & -2 & & 3.52 \\
\hline R temporo-occipital gyrus & $37 / 19$ & 46 & -78 & -16 & & 3.26 \\
\hline L lingual gyrus & 18 & -28 & -88 & -14 & 33 & 3.38 \\
\hline $\mathrm{R}$ fusiform gyrus & 37 & 44 & -50 & -22 & 6 & 2.95 \\
\hline L middle occipital & $37 / 19$ & -40 & -68 & 10 & 18 & 2.94 \\
\hline \multicolumn{7}{|l|}{ B. Isolation - Affiliation } \\
\hline $\mathrm{R}$ cuneus & $17-19$ & 20 & -74 & 46 & 382 & 6.32 \\
\hline L middle occipital & $37 / 19$ & -34 & -88 & 22 & 173 & 5.78 \\
\hline $\mathrm{R}$ fusiform gyrus & 37 & 30 & -42 & -12 & 745 & 5.68 \\
\hline $\mathrm{R}$ cerebellum & & 28 & -44 & -26 & & 5.07 \\
\hline $\mathrm{R}$ cerebellum & & 26 & -58 & -22 & & 4.07 \\
\hline L fusiform gyrus & 37 & -30 & -56 & -16 & 256 & 4.82 \\
\hline L fusiform gyrus & 37 & -30 & -42 & -12 & & 3.7 \\
\hline $\mathrm{R}$ lateral occipital gyrus & $37 / 19$ & 44 & -86 & 14 & 626 & 4.58 \\
\hline $\mathrm{n} / \mathrm{a}$ & & 46 & -98 & 34 & & 3.8 \\
\hline $\mathrm{R}$ supplementary motor area & 6 & 2 & 14 & 54 & 439 & 4.37 \\
\hline $\mathrm{R}$ primary motor cortex & 4 & 6 & 18 & 46 & & 3.56 \\
\hline $\mathrm{R}$ caudal postcentral & 2 & 54 & -28 & 40 & 340 & 4.11 \\
\hline $\mathrm{R}$ caudal postcentral & 2 & 48 & -36 & 46 & & 3.33 \\
\hline $\mathrm{R}$ sup/inf parietal & $7 / 40$ & 36 & -36 & 40 & & 3.02 \\
\hline L supplementary motor & 6 & -24 & 2 & 58 & 37 & 3.75 \\
\hline $\mathrm{R}$ cuneus & 17 & 18 & -54 & 14 & 57 & 3.41 \\
\hline L primary motor & 4 & -38 & -22 & 60 & 26 & 3.27 \\
\hline L cuneus/precuneus & $19 / 7$ & -16 & -80 & 46 & 165 & 3.22 \\
\hline L precuneus & 7 & -6 & -78 & 60 & & 2.9 \\
\hline
\end{tabular}

Note. The data show those regions that emerge in the contrast for both younger and older adults with an overall threshold of $p<.0001$ and an extent threshold of 5 voxels. Up to three local maxima, separated by at least $8 \mathrm{~mm}$, are displayed. Sup $=$ superior; Inf $=$ inferior

signal change in response to affiliation and isolation by each age group. Younger adults showed greater activity than did older adults in higher order visual regions, such as the fusiform gyrus, while older adults showed a greater response than did younger adults in the supplementary motor area (BA 6; Table 4). There was an age-related dissociation based on type of social perception in the paracentral lobule/precuneus, a region that has been implicated in self-referencing and the experience of social pain, and the temporal pole, a region involved in theory of mind. In the paracentral lobule/precuneus, younger adults showed relatively more activity in response to isolation than in response to affiliation, while older adults showed a greater response to affiliation than to isolation (Table 4, Fig. 3b). Interestingly, while distinct regions of the precuneus are recruited for both affiliation and isolation scenes (as discussed previously), these precuneus regions are also recruited differentially by the age groups as a function of the type of social interaction perceived. Within the temporal pole, the opposite pattern was found across the age groups: younger adults showed relatively more activity in response to affiliation than in response to isolation, whereas older adults exhibited greater response when perceiving isolation than when 
Table 4 Age differences in response to social perception as a function of affiliation and isolation

\begin{tabular}{|c|c|c|c|c|c|c|}
\hline Region & $\mathrm{BA}$ & \multicolumn{3}{|c|}{ Activation Peak $(\mathrm{x}, \mathrm{y}, \mathrm{z})$} & \# voxels & $t$ value \\
\hline \multicolumn{7}{|c|}{ A. Younger - Older (Affiliation - Isolation) } \\
\hline $\mathrm{L}$ middle occipital & 18 & -28 & -98 & 2 & 59 & 4.86 \\
\hline $\mathrm{R}$ temporal pole & 38 & 50 & 14 & -34 & 35 & 4.08 \\
\hline $\mathrm{L}$ ventrolateral prefrontal & 47 & -48 & 56 & -6 & 32 & 3.78 \\
\hline L fusiform gyrus & 37 & -28 & -34 & -24 & 12 & 3.76 \\
\hline \multicolumn{7}{|c|}{ B. Older - Younger (Affilation - Isolation) } \\
\hline $\mathrm{R}$ supplementary motor area & 6 & 8 & -2 & 62 & 157 & 5.15 \\
\hline $\mathrm{R}$ superior parietal lobule & 7 & 18 & -46 & 66 & 199 & 4.16 \\
\hline $\mathrm{R}$ paracentral/precuneus & $5 / 7$ & 4 & -38 & 60 & & 4.08 \\
\hline $\mathrm{n} / \mathrm{a}$ & & 44 & -6 & 74 & 17 & 3.94 \\
\hline $\mathrm{L}$ paracentral lobule & 5 & -18 & -10 & 66 & 67 & 3.88 \\
\hline L supplementary motor area & 6 & -8 & 0 & 48 & 29 & 3.84 \\
\hline $\mathrm{L}$ paracentral/precuneus & $5 / 7$ & -10 & -32 & 60 & 15 & 3.61 \\
\hline \multicolumn{7}{|c|}{ C. Younger - Older (Conjunction Affiliation \& Isolation) } \\
\hline R Hippocampus & & 30 & -8 & -18 & 25 & 3.93 \\
\hline
\end{tabular}

Note. The data are thresholded at $p<.001$ (uncorrected) with an extent threshold of 5 voxels. Up to three local maxima, separated by at least 8 mm, are displayed

perceiving affiliation (Table 4, Fig. 3c), which is consistent with our hypotheses based on research demonstrating that older adults show more accurate memory for scenes of isolation than for scenes of affiliation (Gutchess et al., 2012).

\section{Discussion}

In the present study, age-related similarities were found in brain regions associated with theory of mind and self-referencing when affiliation and isolation were perceived. Specifically, perceiving affiliation and isolation scenes similarly engaged the temporal pole, medial prefrontal cortex, and amygdala in younger and older adults. This is in line with our hypotheses and previous literature that has demonstrated a role for the temporal pole in theory of mind (Frith \& Frith, 2003) and the medial prefrontal cortex in self-referencing (Kelley et al., 2002). Age-related preservation has been demonstrated for the temporal pole with respect to theory of mind (Castelli et al., 2010) and for the medial prefrontal cortex in selfreferencing (Gutchess et al., 2007). While we did not have a specific hypothesis for age-related preservation of the amygdala, previous research on theory of mind processing has shown its involvement in detecting emotional meaning from social stimuli such as faces (Adolphs, 1999; reviewed in Siegal \& Varley, 2002). Furthermore, there is evidence for relative agerelated preservation of the amygdala in the emotion perception domain (Kensinger \& Schacter, 2008; Mather et al., 2004).

There were key age-related differences in the neural correlates of perception of affiliation and isolation. Corroborating previous research (Castelli et al., 2010), older adults showed a greater response than did younger adults in a theory of mind region implicated in simulating the experiences of others (Rizzolatti \& Craighero, 2004), the supplementary motor region (BA 6), when perceiving affiliation and isolation. Although not an a priori hypothesis, in response to both affiliation and isolation scenes (vs. nonsocial scenes), younger adults showed greater recruitment of the hippocampus than did older adults, consistent with previous literature demonstrating that younger adults show pronounced activity in the hippocampus, in comparison with older adults, in response to the encoding and retrieval of complex negative and neutral visual scenes (Murty et al., 2009). Age-related differences specific to the type of social interaction emerged in the temporal pole, which is implicated in the retrieval of relevant personal memories and semantic memory scripts that help in the discernment of the thoughts and feelings of others (Frith \& Frith, 2003; Funnell, 2001; Moriguchi et al., 2006), whereby older adults showed a greater response to isolation than to affiliation (while younger adults responded more to affiliation than to isolation). In contrast, in a region relevant to self-referencing, the precuneus, older adults showed a greater response to affiliation than to isolation, but younger adults responded more to isolation than to affiliation.

Age-related differences in the temporal pole in response to affiliation and isolation may reflect differences in preference for the type of social information retrieved from memory in order to understand others' mental states. The temporal pole has been shown to play an integral role in theory of mind (Frith \& Frith, 2003; Moriguchi et al., 2006; reviewed in Olson, Plotzker, \& Ezzyat, 2007). It also is involved in the retrieval of social scripts, since patients with semantic dementia affecting the left temporal pole show great difficulty retrieving scripts (Funnell, 2001), and brain activity in the temporal pole is 
Fig. 3 Age-related differences in response to the conjunction of two types of social perception (affiliation and isolation) were compared (a). MarsBaR region of interest analyses reflect peak activation in each brain region categorized by age group and social perception condition (affiliation, isolation), depicted in bar graphs. Younger adults showed greater activity in the hippocampus, relative to older adults, in response to social (across both affiliation and isolation) versus nonsocial scenes (a). The age differences in response to affiliation versus isolation $(\mathbf{b}, \mathbf{c})$ indicate that in the paracentral lobule and precuneus, younger adults show relatively more activity in response to isolation (relative to affiliation), while older adults show a greater response to affiliation (relative to isolation) (b). In the temporal pole, the opposite pattern is found, with younger adults showing relatively more activity in response to affiliation (relative to isolation), while older adults showed a greater response to isolation (than to affiliation) (c)
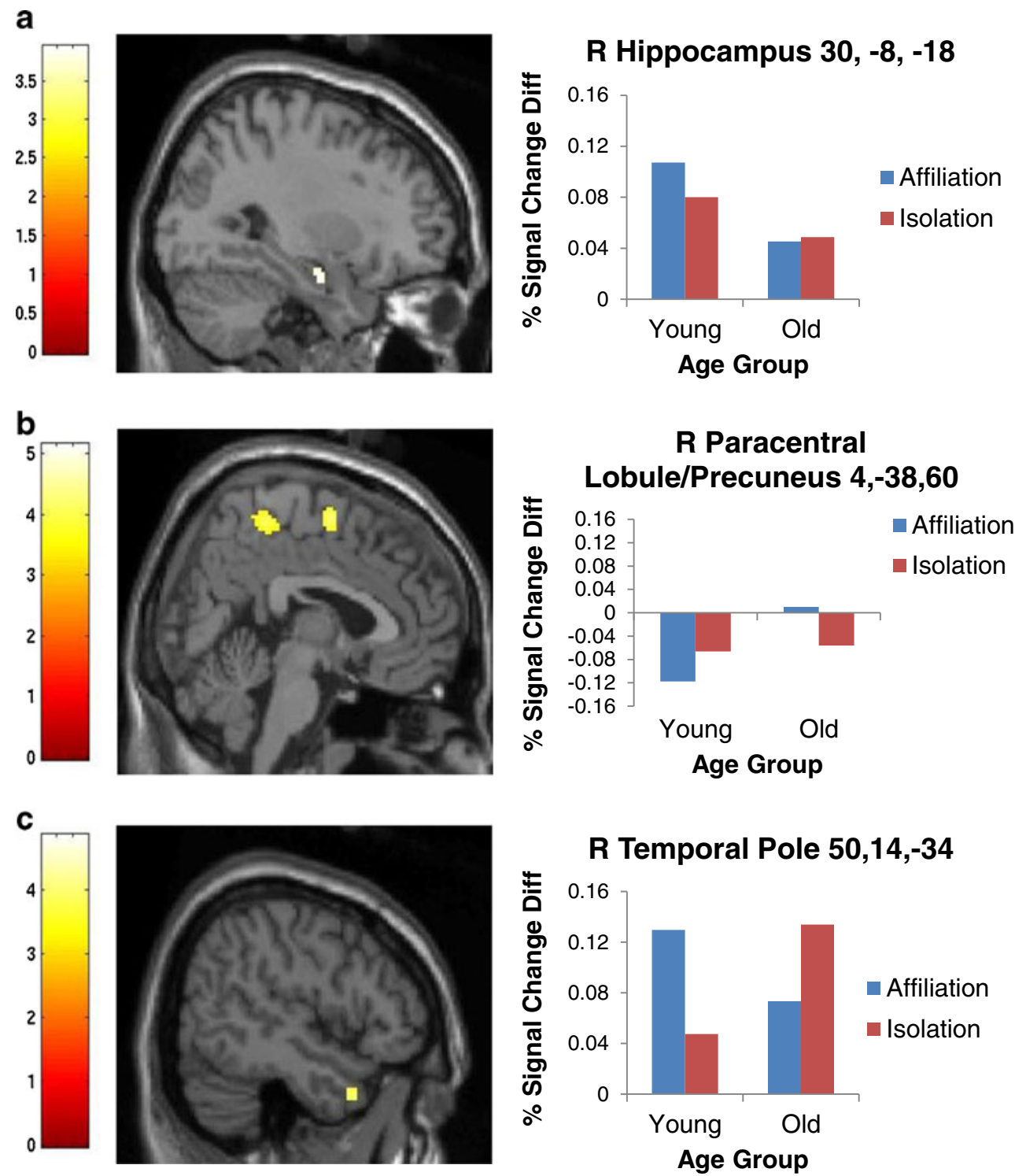

associated with recognition of scripts related to particular social interactions (Moriguchi et al., 2006). Because the temporal pole has been associated both with understanding others' mental states and retrieving relevant personal social knowledge, Moriguchi and colleagues have proposed that its role in theory of mind reflects the utilization of personal knowledge of previous experiences to understand others' mental states (Moriguchi et al., 2006).

Previous research on age-related differences in memory retrieval of affiliation and isolation has shown that older adults have better memory for information about social isolation than for information about affiliation, while younger adults show similar performance for both types of social information (Gutchess et al., 2012). This suggests that the greater recruitment of the temporal pole in older adults in response to social isolation may indicate greater encoding of social isolation into memory, relative to younger adults, as well as the retrieval of personally relevant memories of social isolation to understand others' minds. The finding of greater recruitment of this region in response to affiliation in younger adults may be partially explained by findings in younger adults showing greater recruitment of this region for positive personal memories (vs. negative; Piefke, Weiss, Zilles, Markowitsch, \& Fink, 2003), which may be more similar to the positive social interactions involved in affiliation. Therefore, the temporal pole may be involved in personal memory retrieval relevant to understanding others' minds in both age groups but may specifically respond to situations of social isolation in older adults and affiliation in younger adults.

Age-related differences in recruitment of the precuneus suggest that older adults may engage self-referencing processes to a greater extent in contexts of social affiliation than in isolation. This is in line with evidence that older adults show greater recruitment of brain regions involved in self-referencing 
toward positive than toward negative information (Gutchess et al., 2007; Kensinger \& Schacter, 2008; Leclerc \& Kensinger, 2008; reviewed in Kensinger \& Leclerc, 2009) and, more generally, is consistent with evidence for a positivity effect in older adults (Mather \& Carstensen, 2005). The finding that younger adults showed greater recruitment of this region in response to isolation (than in response to affiliation) may be because this region is also implicated in studies of younger adults examining social exclusion (Eisenberger, Inagaki, Rameson, Mashal, \& Irwin, 2009; Masten et al., 2011), which is similar to the isolation condition in the present study. Furthermore, the precuneus is involved in both the personal experience of pain and perceiving the pain of others (Lamm, Decety, \& Singer, 2011), suggesting functional overlap in selfreferential and other-oriented responses to negative situations such as physical pain. In conclusion, while the precuneus appears to engage self-referencing in both younger and older adults, older adults may respond more to affiliation, while younger adults may respond more to isolation.

The present study has some limitations. The images were not explicitly matched on visual complexity. Although there was an attempt to match the experimental conditions in terms of number of objects/people in each image, neural differences, such as those in the fusiform gyrus, may have emerged due to other differences in visual complexity. In future studies, a more fine-grained matching of visual complexity across conditions will help to reduce potentially spurious brain activations due to differing visual complexity. Our study is novel in that it is the first to measure the neural basis of affiliation and isolation perceptions in older versus younger adults. However, follow-up studies are needed to investigate direct relationships between neural activity in response to affiliation and isolation scenes and behavioral measures of these constructs. For instance, ratings of the degree of affiliation or isolation experienced toward each image could be correlated with brain activity in response to these images. To further examine the components involved in theory of mind and self-referencing with respect to affiliation and isolation, neural activity in response to these images could be associated with an explicit instruction to adopt the mental states of the individuals depicted in these images (vs. a passive viewing condition) or with accuracy of memory for selfrelevant information.

In conclusion, the present study demonstrates both agerelated similarities and differences in the neural basis of affiliation and isolation by implicating regions involved in theory of mind and self-referencing that are differentially activated for older versus younger adults. Building on the present study, future research may specifically elucidate how different types of memory (e.g., semantic and autobiographical memory) interact with theory of mind and selfreferencing processes in response to affiliation and isolation in younger versus older adults.
Author note The authors gratefully acknowledge support from the National Institute on Aging (R21AG032382 to A.H.G.) and the University of Michigan Ross School of Business to C.Y. Postdoctoral fellowship support for J.N.B. was received from T32 Grant NS007292 to the Brandeis University Neuroscience Program. We thank Nicholas Gonzales for experimental assistance.

\section{References}

Adolphs, R. (1999). Social cognition and the human brain. Trends in Cognitive Sciences, 3, 469-479.

Bora, E., Yucel, M., \& Allen, N. B. (2009). Neurobiology of human affiliative behavior: Implications for psychiatric disorders. Current Opinion in Psychiatry, 22, 320-325.

Brett, M., Anton, J. L., Valabregue, R., \& Poline, J. B. (2002, June). Region of interest analysis using an SPM toolbox. Paper presented at the 8th International Conference on Functional Mapping of the Human Brain, Sendai, Japan.

Carstensen, L. L., Isaacowitz, D. M., \& Charles, S. T. (1999). Taking time seriously. A theory of socioemotional selectivity. American Psychologist, 54, 165-181.

Castelli, I., Baglio, F., Blasi, V., Alberoni, M., Falini, A., LivertaSempio, O., ... Marchetti, A. (2010). Effects of aging on mindreading ability through the eyes: An fMRI study. Neuropsychologia, 48, 2586-2594.

Dale, A. M. (1999). Optimal experimental design for event-related fMRI. Human Brain Mapping, 8, 109-114.

Eisenberger, N. I., Inagaki, T. K., Rameson, L. T., Mashal, N. M., \& Irwin, M. R. (2009). An fRMI study of cytokine-induced depressed mood and social pain: The role of sex differences. NeuroImage, 47, 881-890.

Fisher, R. A. (1950). Statistical methods for research workers. London: Oliver \& Boyd.

Folstein, M. F., Folstein, S. E., \& McHugh, P. R. (1975). Mini-mental state: A practical method for grading the cognitive state of patients for the clinician. Journal of Psychiatric Research, 12, $189-198$.

Frith, U., \& Frith, C. D. (2003). Development and neurophysiology of mentalizing. Philosophical Transactions of the Royal Society B, 358, 459-473.

Funnell, E. (2001). Evidence for scripts in semantic dementia: Implications for theories of semantic memory. Cognitive Neuropsychology, 18, 323-341.

Gunning-Dixon, F. M., Gur, R. C., Perkins, A. C., Schroeder, L., Turner, T., Turetsky, B. I., ... Gur, R. E. (2003). Age-related differences in brain activation during emotional face processing. Neurobiology of Aging, 24, 285-295.

Gutchess, A. H., Kensinger, E. A., \& Schacter, D. L. (2007). Aging, self-referencing, and medial prefrontal cortex. Social Neuroscience, 2, 117-133.

Gutchess, A. H., Kensinger, E. A., \& Schacter, D. L. (2010). Functional neuroimaging of self-referential encoding with age. Neuropsychologia, 48, 211-219.

Gutchess, A. H., Yoon, C., \& Schultheiss, O. C. (2012). Implicit motives and memory for social information with age. Unpublished manuscript, Brandeis University.

Hedden, T., Park, D. C., Nisbett, R., Ji, L. J., Jing, Q., \& Jiao, S. (2002). Cultural variation in verbal versus spatial neuropsychological function across the life span. Neuropsychology, 16, 65-73.

Iacoboni, M., Lieberman, M. D., Knowlton, B. J., Molnar-Szakacs, I., Moritz, M., Throop, C. J., \& Fiske, A. P. (2004). Watching social interactions produces dorsomedial prefrontal and medial parietal BOLD fMRI signal increases compared to a resting baseline. NeuroImage, 21, 1167-1173. 
Kaplan, R. M., \& Kronick, R. G. (2006). Marital status and longevity in the United States population. Journal of Epidemiology and Community Health, 60, 760-765.

Kelley, W. M., Macrae, C. N., Wyland, C. L., Caglar, S., Inati, S., \& Heatherton, T. F. (2002). Finding the self? An event-related fMRI study. Journal of Cognitive Neuroscience, 14, 785-794.

Kensinger, E. A., \& Leclerc, C. M. (2009). Age-related changes in the neural mechanisms supporting emotion processing and emotional memory. European Journal of Cognitive Psychology, 21, 192-215.

Kensinger, E. A., \& Schacter, D. L. (2008). Neural processes supporting young and older adults' emotional memories. Journal of Cognitive Neuroscience, 20, 1161-1173.

Koestner, R., \& McClelland, D. C. (1992). The affiliation motive. In C. P. Smith (Ed.), Motivation and personality: Handbook of thematic content analysis (pp. 205-210). New York: Cambridge University Press.

Lamm, C., Decety, J., \& Singer, T. (2011). Meta-analytic evidence for common and distinct neural networks associated with directly experienced pain and empathy for pain. NeuroImage, 54, 24922502.

Lazar, N. A., Luna, B., Sweeney, J. A., \& Eddy, W. F. (2002). Combining brains: A survey of methods for statistical pooling of information. Neurolmage, 16, 538-550.

Leclerc, C. M., \& Kensinger, E. A. (2008). Age-related differences in medial prefrontal activation in response to emotional images. Cognitive, Affective, \& Behavioral Neuroscience, 8, 153-164.

Macrae, C. N., Moran, J. M., Heatherton, T. F., Banfield, J. F., \& Kelley, W. M. (2004). Medial prefrontal activity predicts memory for self. Cerebral Cortex, 14, 647-654.

Masten, C. L., Morelli, S. A., \& Eisenberger, N. I. (2011). An fMRI investigation of empathy for 'social pain' and subsequent prosocial behavior. NeuroImage, 55, 381-388.

Mather, M., Canli, T., English, T., Whitfield, S., Wais, P., Ochsner, K., ... Carstensen, L. L. (2004). Amygdala responses to emotionally valenced stimuli in older and younger adults. Psychological Science, 15, 259-263.

Mather, M., \& Carstensen, L. L. (2005). Aging and motivated cognition: The positivity effect in attention and memory. Trends in Cognitive Sciences, 9, 496-502.

Maylor, E. A., Moulson, J. M., Muncer, A. M., \& Taylor, L. A. (2002). Does performance on theory of mind tasks decline in old age? British Journal of Psychology, 93, 465-485.

Moriguchi, Y., Ohnishi, T., Lane, R. D., Maeda, M., Mori, T., Nemoto, K., ... Komaki, G. (2006). Impaired self-awareness and theory of mind: An fMRI study of mentalizing in alexithymia. NeuroImage, $32,1472-1482$.

Murty, V. P., Sambataro, F., Das, S., Tan, H., Callicott, J. H., Goldberg, T. E., ... Mattay, V. S. (2009). Age-related alterations in simple declarative memory and the effect of negative stimulus valence. Journal of Cognitive Neuroscience, 21, 1920-1933.

Northoff, G., Heinzel, A., de Greck, M., Bermpohl, F., Dobrowolny, H., \& Panksepp, J. (2006). Self-referential processing in our brain -A metaanalysis of imaging studies on the self. NeuroImage, 31, 440-457.

Olson, I. R., Plotzker, A., \& Ezzyat, Y. (2007). The enigmatic temporal pole: A review of findings on social and emotional processing. Brain, 130, 1718-1731.

Piefke, M., Weiss, P. H., Zilles, K., Markowitsch, H. J., \& Fink, G. R. (2003). Differential remoteness and emotional tone modulate the neural correlates of autobiographical memory. Brain, 126, 650-668.

Rizzolatti, G., \& Craighero, L. (2004). The mirror-neuron system. Annual Reviews of Neuroscience, 27, 169-192.

Salthouse, T. A. (1996). The processing-speed theory of adult age differences in cognition. Psychological Review, 103, 403-428.

Salthouse, T. A. (2000). Aging and measures of processing speed. Biological Psychology, 54, 35-54.

Shipley, W. C. (1986). Shipley Institute of living scale. Los Angeles: Western Psychological Services.

Siegal, M., \& Varley, R. (2002). Neural systems involved in 'theory of mind'. Nature Reviews Neuroscience, 3, 463-471.

Slessor, G., Phillips, L. H., \& Bull, R. (2008). Age-related declines in basic social perception: Evidence from tasks assessing eye-gaze processing. Psychology and Aging, 23, 812-822.

Sullivan, S., \& Ruffman, T. (2004). Social understanding: How does it fare with advancing years? British Journal of Psychology, 95, 118

Tessitore, A., Hariri, A. R., Fera, F., Smith, W. G., Das, S., Weinberger, D. R., \& Mattay, V. S. (2005). Functional changes in the activity of brain regions underlying emotion processing in the elderly. Psychiatry Research: Neuroimaging, 139, 9-18.

Vogeley, K., May, M., Ritzl, A., Falkai, P., Zilles, K., \& Fink, G. R. (2004). Neural correlates of first-person perspective as one constituent of human self-consciousness. Journal of Cognitive Neuroscience, $16,817-827$.

Vul, E., Harris, C., Winkielman, P., \& Pashler, H. (2009). Puzzingly high correlations in fMRI studies of emotion, personality, and social cognition. Perspectives on Psychological Science, 4, 274 290.

Wagner, D. D., Kelley, W. M., \& Heatherton, T. F. (2011). Individual differences in the spontaneous recruitment of brain regions supporting mental state understanding when viewing natural social scenes. Cerebral Cortex, 21, 2788-2796.

Wechsler, D. (1997). Wechsler memory scale-third edition. San Antonio, TX: The Psychological Corporation.

Woods, R. P., Cherry, S. R., \& Mazziotta, J. C. (1992). Rapid automated algorithm for aligning and reslicing PET images. Journal of Computer Assisted Tomography, 16, 620-633. 